Objective: A borderline ovarian tumor (BOT) was an important group of tumors of the ovary which needs to be distinguished from invasive ovarian cancer. The most common subtype of BOT in East Asia was the mucinous subtype (mBOT). mBOT was still controversial in terms of management due to different oncologic outcomes and difficulty in preoperative diagnosis. The objectives of the current study were to determine the prognosis and risk factors for recurrence, focusing only on mucinous subtype.

Methods: This retrospective study included patients with mucinous BOT, diagnosed between January 2004 and December 2019, who were operated at Siriraj Hospital. Patients with other subtypes, invasive carcinoma, and other primary carcinomas were excluded.

Results: Two hundred and thirty-five patients were diagnosed as mBOT. The median follow-up time was 54 months. There were 9 patients (3.8\%) who had tumor recurrence. Risk factors of recurrence were a ruptured tumor, presence of ascites, residual tumor, and stage of the tumor. The recurrence rate between fertility-sparing surgery and radical surgery was not different. Detailed surgical staging, a pathological finding of intraepithelial carcinoma, and microinvasion were not associated with recurrence of the disease.

Conclusion: mBOT had an excellent prognosis. Nowadays, fertility-sparing surgery had more roles with no difference in the oncologic outcomes. Patients with risk factors had to be observed closely. Moreover, pre/perioperative diagnosis and accuracy of the final pathological results were also important.

Poster (002)

Epithelial Ovarian Cancer including Borderline Tumor https://doi.org/10.3802/jgo.2021.32.\$1.002

\section{Bowel surgery by gynecologic oncologists during maximal cytoreductive surgery for advanced ovarian cancer}

\section{Chi-Son Chang, Chel Hun Choi, Tae-Joong Kim, Jeong-Won Lee, Byoung-Gie Kim, Yoo-Young Lee*}

Samsung Medical Center, Sungkyunkwan University School of Medicine, Seoul, Korea (yooyoung.lee@samsung.com)

Objective: We report the oncological outcomes in patients with advanced ovarian cancer who had bowel surgery which was performed by gynecologic oncologist (GO) during maximal cytoreductive surgery and compared the outcomes with those of bowel surgery performed by general surgeons (GS).

Methods: Patients who were stage III-IV ovarian cancer and had bowel surgery during maximal cytoreductive surgery were eligible. Patients were divided into two groups according to whether bowel resection was performed by GO (group 1 ) or GS (group 2). In both groups, GO were mainly involved in debulking procedures. Perioperative and survival outcomes were compared between 2 groups.

Results: A total of 268 patients were eligible. Fifty-five patients received bowel surgery by GO (group 1), and 213 patients by GS (group 2). The rate of R0 resection (no residual tumor) after maximal cytoreductive surgery did not differ between 2 groups ( $45.5 \%$ vs. $40.8 \%, \mathrm{p}=0.529$ ). Among extra-uterine procedures, the rate of diaphragm stripping was higher in group $1(72.7 \%$ vs. $40.8 \%, \mathrm{p}<0.001$ ). Operation time (331 minutes [212-608] vs. 362 minutes [173-677], $\mathrm{p}=0.031$ ) and hospital stay (12 days [8-62] vs. 14 days [7-80], $\mathrm{p}<0.001$ ) were slightly longer in group 2. Bowel surgery specific complications did not differ between 2 groups (ileus, $36.4 \%$ vs. $35.7 \%, \mathrm{p}=0.925$; leakage/perforation/ fistula, $3.6 \%$ vs. $6.1 \%$, $\mathrm{p}=0.478$ ). There were no significant differences in progression-free survival and overall survival between 2 groups.

Conclusion: Bowel resection performed by GO was feasible and showed equivalent oncological outcomes when compared with those by GS during maximal cytoreductive surgery for advanced ovarian cancer.

Poster (003)

Imaging in Gynecologic Cancer

https://doi.org/10.3802/jgo.2021.32.S1.003

\section{Comparison of 2D USG based IOTA simple rules and 3D USG in preoperative assessment of adnexal masses}

\section{Rishu Goel, Seema Singhal, J Jyoti Meena, Sunesh Kumar, Smita Manchanda, Kallol Kumar Roy}

All India Institute of Medical Sciences, New Delhi, India (drseemasinghal@gmail.com)

Objective: To compare the diagnostic accuracy of "International Ovarian Tumor Analysis (IOTA) simple rules" and 3-dimensional (3D) ultrasound (USG) to discriminate benign from malignant adnexal masses.

Methods: A prospective observational study was conducted on 84 patients with adnexal masses. Both 2D USG and 3D USG with power doppler were done and IOTA simple rules applied using 2D parameters. Findings were correlated with final histopathology report.

Results: Eighty-four cases were recruited, 41 benign and 43 malignant. IOTA simple rules were conclusive in $88.1 \%$ (74/84) of cases, and the sensitivity and specificity of IOTA simple rules in conclusive cases were $83.78 \%$ (95\% confidence interval $[\mathrm{CI}]=67.99-93.81 \%)$ and $89.19 \%$ (95\% CI=74.58-96.97\%), 